\title{
Pricing conspicuous consumption products in recession periods with uncertain strength
}

\author{
Tony Huschto $\cdot$ Sebastian Sager
}

Received: 6 August 2012/Accepted: 8 January 2014/Published online: 6 February 2014

(c) Springer-Verlag Berlin Heidelberg and EURO - The Association of European Operational Research Societies 2014

\begin{abstract}
We compare different approaches of optimization under uncertainty in the context of pricing strategies for conspicuous consumption products in recession periods of uncertain duration and strength. We consider robust worst-case ideas and how the concepts of Value at Risk (VaR) and Conditional Value at Risk (CVaR) can be incorporated efficiently. The approaches are generic in the sense that they can be applied to other economic decision-making problems with uncertainty. We discuss the strengths and weaknesses of these approaches in general. We quantify runtimes and differences when applied to the special case of pricing decision making. We notice that $\mathrm{VaR}$ results in reliable strategies, although it is not a coherent measure of risk. The $\mathrm{CVaR}$ idea that has become the method of choice in financial mathematics is a very risk-averse version of safeguarding and thus a bit too conservative for pricing decisions. Also the resulting optimal control problem is the most expensive one from a computational point of view. From an economic point of view we observe different safeguarding strategies with respect to when and how prices are adapted. Qualitatively, no surprises arise: The more conservative a strategy is, the sooner prices are reduced to avoid bankruptcy. Yet, the discussion of the advantages and disadvantages is generic and can be transferred to other economic problems. The underlying mathematical model simplifies, as is often the case in economics. In the long run, there is a necessity to consider stochastic processes for the evolvement in time to reduce model-plant mismatch. Thus, our work to understand the behavior of the deterministic model and the impact of different robustification techniques
\end{abstract}

\footnotetext{
T. Huschto $(\bowtie)$

Interdisciplinary Center for Scientific Computing, University of Heidelberg, Heidelberg, Germany e-mail: tony.huschto@iwr.uni-heidelberg.de
}

\section{S. Sager}

Department of Mathematical Optimization, Otto-von-Guericke Universität Magdeburg, Magdeburg, Germany

e-mail: sager@ovgu.de 
should be seen as a step in this direction and as a blueprint for similar decisionmaking tasks with quantitatively verified mathematical models.

Keywords Robust optimal control - Value at risk · Conditional economic system $\cdot$ Recession

\section{Introduction}

Critical economic periods often require firms to react readily and, of course, efficiently. Particular pricing strategies become crucial, especially if the firm has to face a credit crunch recession like the one that started in 2007. In these situations not only the demand is reduced, but the capital markets fail. Accordingly, firms have to self-finance their investments as they cannot borrow money from the market anymore or issue new shares.

Conspicuous consumption products as luxury cars, designer brands, and fancy hotel rooms call for yet additional attention. The demand for those goods is not only characterized by the price, but also reputation plays an important role. Consumers tend to use expensive products to signal and increase their own reputation (Nelissen and Meijers 2011):

"Why are people so keen on wearing brand-labeled clothes and owning other luxury-branded products to pay a premium for them?

The answer appears to be to gain social status."

Amaldoss and Jain (2005a, b), Kort et al. (2006), Caulkins et al. (2010, 2011) extensively analyzed the problem of pricing conspicuous goods. The firm's manager always has to balance prices between keeping the future demand at a high level by charging high prices and preventing bankruptcy as high prices reduce the depleted demand even more. This, in turn, can cause negative cash levels that are tantamount to bankruptcy in situations when no money is available at the capital markets.

In Huschto et al. (2011) we extended the aforementioned results by establishing a new numerical methodology based on a scenario tree formulation and the introduction of a delayed effect of the current price on the reputation. This implies that price changes have a delayed effect on the consumer's reaction as they have to get accustomed to the new situation.

While the duration of the economic crisis has been considered as a random variable by Caulkins et al. $(2010,2011)$ and Huschto et al. (2011), its strength was assumed to be known. In fact, it has been deduced that one has to distinguish three major types of recession, depending on the rate of the demand reduction within the recession period: mild, intermediate, and severe crises. These are mainly characterized by the corresponding bankruptcy probabilities and require different pricing strategies and initial levels of reputation and cash.

Still, in real world scenarios one does not know the strength of the recession beforehand, often firms have to deal with this special situation while it is already apparent. To gain more insight into the effects of uncertain recession strength, the 
present paper regards it as a random variable as well, addressing the induced problem by various probabilistic approaches.

Thereby, we consider (robust) worst-case investigations first. Those are typically used in chemical engineering, e.g., to avoid irreversible reactions in runaway processes (Kühl et al. 2007). Bertsimas et al. (2011) give a broad overview over current research in robust optimization, focusing on modeling ideas, some practical applications, and demarcating it from stochastic optimization approaches (Prékopa 1995; Shapiro et al. 2009). Furthermore, the ideas in Bertsimas et al. (2011) are guided by assuming the included uncertainty to be set based. Thus one wants to deduce solutions that are feasible for any realization of the uncertainty within this given set, which is the only reasonable alternative if there exists no distributional information about the parameter uncertainty.

But especially in economic settings decision makers do not want to target worst possible outcomes, but rather more likely scenarios. Bertsimas et al. (2011) characterize these probabilistic guarantees by classifying the uncertainty sets differently, introducing a budget of uncertainty. In the same context the quantification of risk (Rockafellar 2007) becomes necessary and the conspicuous good's manager has to base his strategies on different measures, e.g., probabilities of compliance or coherent risk measures, to price the product appropriately taking an acceptable risk.

To that end, we implement and compare the robust worst-case approaches with ideas of Value at Risk (VaR) and Conditional Value at Risk (CVaR). Therefore, we present adequate reformulations needed for incorporating these probabilistic constraints within the original optimal control problem. For the first time, we analyze the strengths and weaknesses of all considered approaches from a numerical and economical point of view in the special context of the conspicuous consumption model. Furthermore, we quantify the differences between these approaches to optimization under uncertainty by comparing their optimal performance.

Moreover, we observe that the price reductions that are necessary to survive a recession with uncertain strength depending on a desired confidence level are optimally conducted by decreasing prices adaptively with the actual duration of the crisis rather than choosing a fixed (reduced) price for the overall recession, as it is known from feedback characteristics of stochastic control.

The paper is organized as follows. In Section 2 we recall the underlying model and present the emerging optimal control problem. In Sects. 3 and 4 we introduce the different measures of risk treated and apply them to our application. This includes robust worst-case approaches approximated by a linearization idea (Diehl et al. 2006) and the sigmapoint approach (Recker et al. 2011), as well as the VaR and CVaR. After discussing the advantages and disadvantages of the mentioned approaches both in the context of economics and from a numerical point of view in Sect. 5, in Sect. 6 numerical results and their economic interpretations illustrate the presented ideas. We conclude by summarizing the results and giving an outlook on future research in Sect. 7. 


\section{The underlying optimal control problem}

\subsection{Model formulation}

Our basic problem, as analyzed and discussed in detail in , is based on an economic setting with a recession period followed by a normal economic period, compare Fig. 1. The value $\tau$ denotes the endpoint of the crisis.

The dynamics of our model includes two states. The brand image $A$, representing the exclusiveness of the product, evolves in both periods proportional to the price $p$, which is the control of our problem. In the context of exclusive goods, higher prices work positively on demand. The available cash $B$ becomes crucial in situations when the capital markets cease to function. Then firms have to budget with their reserves as they cannot borrow money from or issue new shares to the market. The cash $B$ depends on the gains $p(\cdot) \cdot D(\cdot)$ of the firm, fixed costs $C$, and the short-time interest $\delta$. Therein the demand $D$ is driven by the brand image and the pricing strategy $p$. It is essentially influenced by the economic stage, i.e., in the normal period $(N)$ we have

$$
D_{\mathrm{N}}(A(t), p(t))=m-\frac{p(t)}{A(t)^{\beta}},
$$

whereas in the recession $(R)$ demand is reduced to

$$
D_{\mathrm{R}}(A(t), p(t))=D_{\mathrm{N}}(A(t), p(t))-\alpha .
$$

The positive parameter $\alpha$ measures the strength of the crisis, which we assume to be constant in time. The parameter $0<\beta<1$ is given and $m$ corresponds to the potential market size.

The objective of the company is to maximize the expected value of profit over the finite or infinite time horizon $\left[0, t_{\mathrm{f}}\right]$ of interest. The profit is composed of two parts: the gains of the normal economic period $\left(\tau, t_{\mathrm{f}}\right]$ and an impulse dividend of the cash reserve at the end of the recession phase, $B(\tau)$. This dividend is included as the capital market is assumed to become functional again in the normal economic period and firms can freely borrow and lend cash then. Thus, the firm does not need a positive cash level $B(\cdot)$ on $\left(\tau, t_{\mathrm{f}}\right]$. For a fixed $\tau$ and a given discount rate $r$, the objective function is calculated as

$$
\Phi(\tau)=\mathrm{e}^{-r \tau} B(\tau)+\int_{\tau}^{t_{\mathrm{f}}} \mathrm{e}^{-r t}\left(p(t) D_{\mathrm{N}}(A(t), p(t))-C\right) \mathrm{d} t
$$

being the sum of these two components, resulting in the optimal control problem

Fig. 1 Stages $\left[t_{0}, \tau\right]$ and $\left[\tau, t_{\mathrm{f}}\right]$ of the recession model

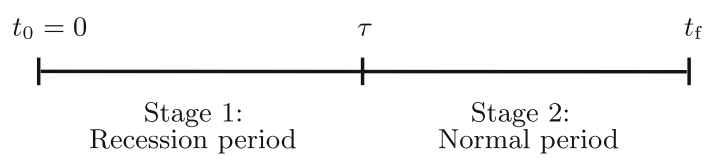




$$
\begin{array}{rlrl}
\max _{p(\cdot)} \Phi(\tau) & & \\
\text { s.t. } & \dot{A}(t) & =\kappa(\gamma p(t)-A(t)), & \\
\dot{B}(t) & =p(t) D_{\mathrm{R}}(A(t), p(t))-C+\delta B(t), & & t \in\left[0, t_{\mathrm{f}}\right], \\
& A(0) & =A_{0}, \quad B(0)=B_{0}, & \\
0 & \leq D_{\mathrm{R} / \mathrm{N}}(A(t), p(t)), & & \\
p(t) & \geq 0, & & t \in\left[0, t_{\mathrm{f}}\right], \\
B(t) & \geq 0, & & t \in\left[0, t_{\mathrm{f}}\right],
\end{array}
$$

with $D_{\mathrm{R} / \mathrm{N}}(A(t), p(t))$ given as in (1) and $B(t)$ negligible in the normal period $\left(\tau, t_{\mathrm{f}}\right]$. The parameters $\kappa$ and $\gamma$ denote positive scaling parameters in the context of the brand image and $A_{0}$ and $B_{0}$ are the initial values of brand image and cash reserve, respectively. However, typically the recession length $\tau$ is not known beforehand to decision makers. An individual firm also has no influence on when the recession ends. Therefore, we assume that the length of the recession period $\tau$ is an exponentially distributed random variable, consistent with the discussions of Caulkins et al. (2011). The goal changes to maximizing the expectation value of the net present value (NPV) at time $\tau$, i.e., the objective function $\Phi$ weighted by the exponential probability density function with rate parameter $\lambda$,

$$
\max _{p(\cdot)} \mathbb{E}[\operatorname{NPV}(\tau)]:=\max _{p(\cdot)} \int_{0}^{t_{\mathrm{f}}} \lambda \mathrm{e}^{-\lambda \tau} \Phi(\tau) \mathrm{d} \tau
$$

subject to the constraints given in (3) for all $0 \leq \tau \leq t_{\mathrm{f}}$.

In the following sections we regard an additional source of uncertainty. As the recession strength $\alpha$ is likewise unknown to decision makers beforehand, we assume it to be a random variable as well, which requires the use of probabilistic constraints. We give a detailed discussion on the different modeling ideas and reformulations in the corresponding Sects. 3 and 4.

The emerging problem is a non-standard optimal control problem in the sense that different kinds of uncertainty are present, making analytical investigations difficult. Therefore, we propose a numerical result-driven approach.

\subsection{Numerical treatment}

We propose to use reformulations to transfer the optimal control problem (4) into a more standard form that can be efficiently solved. By discretizing the uncertainty in the exponentially distributed recession length $\tau$ we can use a scenario tree approach to deduce a multi-stage, nonlinear optimal control problem that can be tackled by numerical methods such as Bock's direct multiple shooting approach (Bock and Plitt 1984; Leineweber 1999; Leineweber et al. 2003).

Following the ideas introduced in Huschto et al. (2011), we obtain a multi-stage optimal control problem that depends on the number of discretized recession ends 
$\tau_{i}, i=1, \ldots, n$, the appropriate objective functions $\Phi_{i}$ with corresponding probabilities $\mathbb{P}_{i}$ (here, as multiplicative factors), as well as transition functions $f_{\operatorname{tr} A, i}, f_{\operatorname{tr} B, i}$ and equality constraints $r_{\mathrm{eq}, i}$ to guarantee correct initializations of the state variables $A_{i}, B_{i}$ in the resulting $n$ recession and $n$ normal period stages.

$$
\begin{array}{rlrl}
\max _{p(\cdot)} \sum_{i=1}^{n} \Phi_{i}\left(\tau_{i}, A_{n+i}(t), B_{i-1}\left(\tau_{i}\right), p(t), \mathbb{P}_{i}\right) & & \\
\text { s.t. } \quad \dot{A}_{i}(t) & =\kappa\left(\gamma p(t)-A_{i}(t)\right), & & \\
\dot{B}_{i}(t) & =p(t) D_{\mathrm{R}}\left(A_{i}(t), p(t)\right)-C+\delta B_{i}(t), & & t \in\left[0, \tau_{i}\right], \quad 0 \leq i \leq n-1, \\
A_{0}(0) & =A_{0}, \quad B_{0}(0)=B_{0}, & & \\
0 & \leq D_{\mathrm{R}, \mathrm{N}}\left(A_{i}(t), p(t)\right), & & t \in\left[0, t_{\mathrm{f}}\right], \\
p(t) & \geq 0, & t \in\left[0, t_{\mathrm{f}}\right], & \\
B_{i}(t) & \geq 0, & t \in\left[0, \tau_{i}\right], 1 \leq i \leq n-1, \\
A_{i}\left(\tau_{i}\right) & =f_{\operatorname{tr} A, i}\left(A_{i-1}\left(\tau_{i}\right)\right), & & \\
B_{i}\left(\tau_{i}\right) & =f_{\operatorname{tr} B, i}\left(B_{i-1}\left(\tau_{i}\right)\right), & & 1 \leq i \leq n \\
0 & =r_{\mathrm{eq}, i}\left(A_{i}\left(t_{i-n}\right), A_{i-n-1}\left(\tau_{i-n}\right)\right), & & 1 \leq i \leq n-1,
\end{array}
$$

\section{Measures of risk in optimal control with uncertainties}

In optimization we often have to deal with situations, where the problem is affected by external disturbances or, like in our current recession period model, by uncertainties in the parameters. These influences may cause different or even critical results when applying controls of the undisturbed problem in reality. Therefore, alternative controls have to be determined to guarantee a certain robustness against the uncertainties.

Before we study important types of risk measures and their consequences for optimal control, let us consider an optimal control problem of the form

$$
\begin{array}{rlr}
\min _{x, u} \Phi(x, u ; p) & \\
\text { s.t. } \quad \dot{x}(t) & =f(x(t), u(t) ; p), \quad t \in[0, T], \\
x(0) & =x_{0}, \\
0 & \geq c(x(t), u(t) ; p), \quad t \in[0, T],
\end{array}
$$

with state variables $x \in \mathbb{R}^{n_{x}}$, controls $u \in \mathbb{R}^{n_{u}}$, and model parameters $p \in \mathbb{R}^{n_{p}}$. The smooth real valued functions $\Phi, f, c$ characterize the objective (6a), state dynamics defined by the ordinary differential equation (ODE) (6b) with initial values (6c), and path constraints $(6 \mathrm{~d})$, respectively. In the rest of this paper, the constraints $c(\cdot)$ are the subject of special interest, as we have to stipulate to what extent they should be 
satisfied if uncertainty in the parameters $p$ is present. Naturally the objective $\Phi(\cdot)$ can comprise a related dependency. However, on one hand we can transfer the uncertainty included in the objective to an additional constraint, leaving the resulting objective unaffected by uncertain parameters. On the other hand, we address certain formulations of the performance criterion determined by economic considerations later on.

In the following subsections, we take a closer look upon (worst-case) robust optimal control approaches and the concepts of $\mathrm{VaR}$ and $\mathrm{CVaR}$. In doing so we review methods on how to incorporate the different probabilistic constraints into an underlying optimal control problem and how to obtain or approximate a solution to the emerging problem. Thereafter, we apply the concepts on the economic consumption problem and focus on the strengths and weaknesses of the different approaches, both from an economic and numerical point of view.

\subsection{Robust approaches}

The basic idea of a robust optimization of problem (6) is to find controls $u(\cdot)$ that fulfill the constraints $c(\cdot)$ within a whole region of confidence. Assuming that we know that the parameters are Gaussian with expectation $\bar{p}$ and covariance matrix $\Sigma$, then depending on the desired confidence level $\omega>0$ the constraints become

$$
0 \geq \max _{\|p-\bar{p}\|_{2, \Sigma^{-1}} \leq \omega} c(x(t), u(t) ; p), \quad t \in[0, T] .
$$

This formulation clearly includes the traditional worst-case analysis, where the goal is to eliminate all risk, but also more general uncertainty sets can be included in the optimization problem.

\subsubsection{Linearization}

An idea to approximate the robust problem with constraint (7) was proposed by Ma and Braatz (2001), Nagy and Braatz (2004), Diehl et al. (2006). If the constraint functions are monotone within the parameter set and can be approximated by a suitable Taylor expansion, one can show that up to first order we have

$$
\max _{\|p-\bar{p}\|_{2, \Sigma^{-1}} \leq \omega} c(x, u ; p) \approx c(x, u ; \bar{p})+\omega\left\|\frac{\mathrm{d}}{\mathrm{d} p} c(x, u ; p)\right\|_{2, \Sigma}
$$

with the notation from before. Thus, we can reformulate the given optimal control problem by replacing the constraint (7) by the linearization (8). The remaining question is how to deal with the uncertainty within the objective $\Phi$. The most common variants are inserting the nominal value $\bar{p}$, i.e.,

$$
\mathcal{Z}(x, u ; p)=\Phi(x, u ; \bar{p}),
$$

relying on an expectation

$$
\mathcal{Z}(x, u ; p)=\mathbb{E}[\Phi(x, u ; p)],
$$


or using the measure already applied to the constraint. In the current approach, this results in optimizing over a (possibly different) probability set depending on the characteristics of the random parameter to a confidence level $\omega_{0}$.

Then we can finally reformulate the original optimal control problem as

$$
\begin{array}{cll}
\min _{x, u} & \mathcal{Z}(x, u ; p) & \\
\mathrm{s.t.} & \dot{x}(t)=f(x(t), u(t) ; p), & t \in[0, T], \\
& x(0)=x_{0}, & \\
& 0 \geq c(x(t), u(t) ; \bar{p})+\omega\left\|\frac{\mathrm{d}}{\mathrm{d} p} c(x(t), u(t) ; p)\right\|_{2, \Sigma}, & t \in[0, T] .
\end{array}
$$

\subsubsection{The sigmapoint approach}

An alternative approach to solve robust optimal control problems was proposed in Recker et al. (2011). It is based on the Unscented Transformation technique (Julier and Uhlmann 1996; Heine et al. 2006) for propagating distributed information through given nonlinear models. This idea allows to combine a moderate computational effort of the linearized worst-case formulation with the higher accuracy of, e.g., a high-order Taylor approximation of the constraint (7). The fundamental idea of the unscented transformation is to choose modified constraints $\tilde{c}(x, u ; p)$ such that satisfying these new constraints results in satisfying the original constraints $c(x, u ; p)$ for all parameters $p$ within the critical subspace for a given probability level $\zeta$, i.e.,

$$
\tilde{c}(x, u ; p) \leq 0 \quad \Rightarrow \quad \mathbb{P}[c(x, u ; p) \leq 0] \geq \zeta .
$$

Possible choices of the modified constraints are the principal axis endpoints of the constraint distribution. But to identify these endpoints, the mapping of the parameter distribution onto the constraints has to be known, which is often difficult. Hence, a remedy to this was presented by proposing to use so-called sigmapoints with corresponding weights and propagate these through the underlying model. If the weighted sigmapoints approximate the distribution of the parameters $p$ one can approximate the distribution of the constraints by that means (Julier and Uhlmann 1996). As Julier and Uhlmann (1997) showed, this allows to match the first two moments of the constraint distribution exactly.

One choice for choosing the modified constraints $\tilde{c}$ (using parameters that are normally distributed) is

$$
\tilde{c}\left(x, u ; p_{i}\right)=c(x, u ; \bar{p})+\omega\left\|c(x, u ; \bar{p})-c\left(x, u ; p_{i}\right)\right\|_{2}, \quad i=0, \ldots, 2 n_{p},
$$

with the sigmapoints

$$
\begin{gathered}
p_{0}=\bar{p}, \\
p_{i}=\bar{p}+\sqrt{\Sigma_{i}}, \quad i=1, \ldots, n_{p}, \\
p_{n_{p}+i}=\bar{p}-\sqrt{\Sigma_{i}}, \quad i=1, \ldots, n_{p},
\end{gathered}
$$

(Recker et al. 2011). Therein, $\bar{p}$ is the set of nominal parameters of dimension $n_{p}$ and $\Sigma_{i}$ is the $i$-th row or column of the covariance matrix $\Sigma$. 
For not normally distributed parameters the resulting approximation of the constraint distribution may be erroneous, which can cause bad approximations of the robust solutions. Still, industrial applications (Recker et al. 2011) have shown that using modified constraints

$$
\tilde{c}\left(x, u ; p_{i}\right)=c\left(x, u ; p_{i}\right)
$$

with the sigmapoints defined as in (10b-10d) instead of (10a) leads to reasonable approximations, even if the parameters are not normally distributed.

Thus, the resulting robust optimal control problem becomes

$$
\begin{array}{cl}
\min _{x, u} & \mathcal{Z}(x, u ; p) \\
\mathrm{s.t.} & \dot{x}(t)=f(x(t), u(t) ; p), \quad t \in[0, T], \\
& x(0)=x_{0}, \\
& 0 \geq \tilde{c}\left(x(t), u(t) ; p_{i}\right), \quad t \in[0, T], \quad i=0, \ldots, 2 n_{p},
\end{array}
$$

where the objective function $\mathcal{Z}$ is given as in the previous section, including possible formulations based on the introduced sigmapoints.

\subsection{Value at risk}

As an alternative to robust optimization we consider chance constraints. Hence, we require the constraints $c(\cdot) \leq 0$ to be satisfied only with a given probability $\zeta$. Such a formulation is identical to the VaR (Artzner et al. 1999; Jorion 2006).

Definition 1 For a random variable $X$ with cumulative distribution function $F_{X}$ and a given probability level $\zeta \in(0,1)$ the $\mathrm{VaR}$ of $X$ is given by

$$
\operatorname{VaR}_{\zeta}(X)=q_{\zeta}(X)=\min _{z}\left\{F_{X}(z) \geq \zeta\right\}
$$

Therein, $q_{\zeta}$ denotes the $\zeta$-quantile of $X$, which is equal to the VaR. Hence, if we pass to a chance constraint for the original constraint $c(\cdot) \leq 0$, the following relation holds true.

$$
\mathbb{P}[c(x, u ; p) \leq 0] \geq \zeta \quad \Leftrightarrow \quad q_{\zeta}(c(x, u ; p)) \leq 0 \quad \Leftrightarrow \quad \operatorname{VaR}_{\zeta}(c(x, u ; p)) \leq 0 .
$$

Incorporating this into our optimal control problem (6), we obtain the safeguarding problem with VaR constraint

$$
\begin{array}{cll}
\min _{x, u} & \mathcal{Z}(x, u ; p) & \\
\mathrm{s.t.} & \dot{x}(t)=f(x(t), u(t) ; p), & t \in[0, T], \\
& x(0)=x_{0}, & \\
& 0 \geq \operatorname{VaR}_{\zeta}(c(x(t), u(t) ; p)), & t \in[0, T],
\end{array}
$$

where $\bar{p}$ denotes again the nominal value and $\zeta$ the desired probability level. Certainly, one can use the VaR formulation (with a different probability level $\zeta^{\prime}$ ) for the objective function as well rather than keeping with the nominal value or an expectation value. 
Similar to the previous subsection, the implementation of the VaR constraint in problem (15) requires knowing the distribution of the constraint $c$ depending on the variable $p$. For constraints depending on only one uncertain parameter we obtain the following useful reformulation.

Theorem 1 If the constraint function $c(\cdot)$ is a smooth function of the parameter $p$ and monotone in $p$, then

$$
\operatorname{VaR}_{\zeta}(c(\cdot ; p)) \leq 0 \quad \Leftrightarrow \quad \mathbb{P}[c(\cdot ; p) \leq 0] \geq \zeta \quad \Leftrightarrow \quad c\left(\cdot ; \operatorname{VaR}_{\zeta}(p)\right) \leq 0 .
$$

Proof We can easily calculate

$$
\begin{aligned}
\mathbb{P}[c(\cdot ; p) \leq 0] \geq \zeta & \Leftrightarrow \mathbb{P}\left[p \leq c^{-1}(0)\right] \geq \zeta \\
& \Leftrightarrow \quad \operatorname{VaR}_{\zeta}(p) \leq c^{-1}(0) \\
& \Leftrightarrow \quad c\left(\cdot ; \operatorname{VaR}_{\zeta}(p)\right) \leq 0,
\end{aligned}
$$

where $c^{-1}(\cdot)$ denotes the inverse function of $c(\cdot)$ with respect to the independent variable $p$.

\subsection{Conditional value at risk}

Based on the theoretical weaknesses of the VaR the sophisticated $\mathrm{CVaR}$ has been introduced by Rockafellar and Uryasev (2000, 2002). Given a random variable $X$ with respect to a probability level $\zeta$, they describe the CVaR as the expectation of $X$ in conditional distribution of its upper $\zeta$-tail. That means that $\mathrm{CVaR}$, setting it apart from the traditional $\mathrm{VaR}$, regards not only the occurrence of negative outcomes (or losses), but also their extent (or amount). This always yields the CVaR to be a more cautious risk measure than the $\mathrm{VaR}$.

Definition 2 (Acerbi 2002) The Conditional Value at Risk of a random variable $X$ is given as

$$
\operatorname{CVaR}_{\zeta}(X)=\frac{1}{1-\zeta} \int_{\zeta}^{1} \operatorname{VaR}_{z}(X) \mathrm{d} z
$$

The definition confirms the above statement directly. As a more applicationoriented version, Rockafellar and Uryasev (2000, 2002) established the following.

Definition 3 For a random variable $X$ we obtain the Conditional Value at Risk by the minimization formula

$$
\operatorname{CVaR}_{\zeta}(X)=\min _{\vartheta \in \mathbb{R}}\left\{\vartheta+\frac{1}{1-\zeta} \mathbb{E}[\max \{0, X-\vartheta\}]\right\} .
$$

This term leads to an important connection to the VaR risk again [apart from the one given by (17)], i.e., (Rockafellar 2007) 


$$
\operatorname{VaR}_{\zeta}(X)=\text { left endpoint of } \arg \min _{\vartheta \in \mathbb{R}}\left\{\vartheta+\frac{1}{1-\zeta} \mathbb{E}[\max \{0, X-\vartheta\}]\right\} \text {. }
$$

For practical applications, another property of CVaR has shown to be important.

Theorem 2 (Rockafellar 2007) The Conditional Value at Risk of a random variable $X \in \mathcal{L}^{2}$ depends continuously on the probability level $\zeta \in(0,1)$ and has the limits

$$
\lim _{\zeta \rightarrow 0} \mathrm{CVaR}_{\zeta}(X)=\mathbb{E}[X] \text { and } \lim _{\zeta \rightarrow 1} \mathrm{CVaR}_{\zeta}(X)=\sup X
$$

Remark 1 Note that for $\zeta \rightarrow 1$ the $\operatorname{VaR}_{\zeta}(X)$ tends to $\sup X$ as well, but it does not tend to $\mathbb{E}[X]$ for $\zeta \rightarrow 0$. To check this, consider the quantiles of the normally distributed random variable $Z \sim \mathcal{N}(\mu, \sigma)$. It holds $\lim _{\zeta \rightarrow 0} q_{\zeta}(Z)=-\infty$.

Safeguarding a robust optimal control problem with CVaR constraints can be formalized analogous to the approach before. We obtain the new problem

$$
\begin{array}{cll}
\min _{x, u} & \mathcal{Z}(x, u ; p) & \\
\mathrm{s.t.} & \dot{x}(t)=f(x(t), u(t) ; p), & t \in[0, T], \\
& x(0)=x_{0} & \\
& 0 \geq \mathrm{CVaR}_{\zeta}(c(x(t), u(t) ; p)), & t \in[0, T] .
\end{array}
$$

By the minimization rule (18) these constraints can be readily implemented into the original problem, without extra care of the VaR or the distribution of the constraint function $c(\cdot)$ depending on the parameter. Nevertheless, the emerging $\mathrm{CVaR}$ constraint $0 \geq \mathrm{CVaR}_{\zeta}(c(x, u ; p))$ has to be fulfilled within the whole time interval $[0, T]$. Thus the control parameter $\vartheta$ of (18) has to be chosen for every $t \in[0, T]$ and we obtain a control function $\vartheta(\cdot)$ in that context.

Finally, due to Theorem 2 the analysis of expectation-based and worst-case approaches can be performed in the context of CVaR as well.

\section{Application to the conspicuous consumption model}

In the conspicuous consumption problem (3) we consider the recession strength $\alpha$ as an additional source of uncertainty. Decision makers do not know the actual magnitude of the crisis before they really have to face it. Hence, they have to apply pricing strategies that are in some sense robust against the real strength to avoid bankruptcy of the firm. Consequently, the bankruptcy constraint $B(t ; \alpha) \geq 0$ [compare $(3 \mathrm{~g})$ ] becomes probabilistic now and can be treated by the aforementioned approaches. Furthermore, one has to adapt the objective function, namely the maximization of the profit function (4), to the new situation.

A reasonable choice from an economic point of view is to reduce prices at the beginning of the crisis such that the company can cope with an average-heavy recession indicated by a certain $\hat{\alpha}$, i.e., using the objective function [compare (2)] 


$$
\Phi^{1}(\tau, \hat{\alpha})=\mathrm{e}^{-r \tau} B(\tau, \hat{\alpha})+\int_{\tau}^{t_{\mathrm{f}}} \mathrm{e}^{-r t}\left(p(t) D_{\mathrm{N}}(A(t), p(t))-C\right) \mathrm{d} t .
$$

Then prices can be reduced further if the crisis turns out to be more severe than expected first. The big disadvantage of that objective is that if the actual $\alpha$ is smaller than anticipated, the firm cannot increase profit by setting higher prices as those are not optimal for the given objective function. Hence to include the possibility of setting the highest possible price, our objective should be based on the situation where we have no recession, i.e.,

$$
\Phi^{2}(\tau, \alpha=0)=\mathrm{e}^{-r \tau} B(\tau, 0)+\int_{\tau}^{t_{\mathrm{f}}} \mathrm{e}^{-r t}\left(p(t) D_{\mathrm{N}}(A(t), p(t))-C\right) \mathrm{d} t .
$$

Then the reduction of prices during the recession of strength $\alpha$ is only depending on the probabilistic constraint $B(t ; \alpha) \geq 0$ being active.

\subsection{Linearization}

The linearization approach (and the sigmapoint idea) introduced in the previous section depends on normally distributed parameters. Let $\alpha$ be a Gaussian random variable truncated to the interval $0 \leq \alpha \leq m$ with mean value $\bar{\alpha}$ and variance $\Sigma$. The truncation becomes necessary to guarantee that the constraint for the demand to be positive can be satisfied for all realizations of $\alpha$. However, for our choices of meanvariance combinations (compare Sect. 6) the differences to a standard normal distribution are neglectable.

Then the original bankruptcy constraint $B(t) \geq 0$ is replaced as in (9) to obtain

$$
0 \leq B(t ; \bar{\alpha})+\omega\left\|\frac{\mathrm{d}}{\mathrm{d} \alpha} B(t ; \alpha)\right\|_{2, \Sigma} .
$$

Thus, the constraint depends on the choices of the desired probability level $\omega$ and the variance $\Sigma$. Still, if the variance of the random parameter is not given but object of our investigation we can fix the probability level to, say, $\omega=1$ and consider only different values of $\Sigma$ indicating a combination of both notions.

The resulting problem reads $(i=1,2)$

$$
\begin{array}{cll}
\max _{p(\cdot)} & \mathbb{E}\left[\Phi^{i}(\tau)\right] & \\
\text { s.t. } & \dot{A}(t)=\kappa(\gamma p(t)-A(t)), & t \in\left[0, t_{\mathrm{f}}\right], \\
& \dot{B}(t)=p(t) D_{\mathrm{R}}(A(t), p(t))-C+\delta B(t), & t \in[0, \tau], \\
& A(0)=A_{0}, \quad B(0)=B_{0}, & \\
& 0 \leq D_{\mathrm{R} / \mathrm{N}}(A(t), p(t)), & t \in\left[0, t_{\mathrm{f}}\right], \\
& p(t) \geq 0, & t \in\left[0, t_{\mathrm{f}}\right], \\
& 0 \leq B(t ; \bar{\alpha})+\omega\left\|\frac{\mathrm{d}}{\mathrm{d} \alpha} B(t ; \alpha)\right\|_{2, \Sigma}, & t \in[0, \tau],
\end{array}
$$

with $D_{\mathrm{R} / \mathrm{N}}$ as in (1) and $\Phi^{i}, i=1,2$, denoting the objective function as in (22a) and (22b), respectively. 


\subsection{Sigmapoint approach}

For $\alpha$ having a truncated normal distribution on the interval $0 \leq \alpha \leq m$ with mean $\bar{\alpha}$ and variance $\Sigma$, we use the sigmapoints

$$
\begin{aligned}
& \alpha_{0}=\bar{\alpha} \\
& \alpha_{1}=\bar{\alpha}+\sqrt{\Sigma} \\
& \alpha_{2}=\bar{\alpha}-\sqrt{\Sigma}
\end{aligned}
$$

and the modified constraint

$$
\tilde{B}\left(t ; \alpha_{i}\right)=B\left(t ; \alpha_{i}\right), \quad i=0,1,2 .
$$

The emerging robust optimal control problem becomes

$$
\begin{aligned}
& \max _{p(\cdot)} \mathbb{E}\left[\Phi^{i}(\tau)\right] \\
& \text { s.t. } \quad \dot{A}(t)=\kappa(\gamma p(t)-A(t)), \quad t \in\left[0, t_{\mathrm{f}}\right], \\
& \dot{B}(t)=p(t) D_{\mathrm{R}}(A(t), p(t))-C+\delta B(t), \quad t \in[0, \tau], \\
& A(0)=A_{0}, B(0)=B_{0} \text {, } \\
& 0 \leq D_{\mathrm{R} / \mathrm{N}}(A(t), p(t)), \quad t \in\left[0, t_{\mathrm{f}}\right], \\
& p(t) \geq 0, \quad t \in\left[0, t_{\mathrm{f}}\right], \\
& 0 \leq B\left(t ; \alpha_{j}\right), \quad t \in[0, \tau], \quad j=0,1,2 \text {, }
\end{aligned}
$$

with the notations as introduced before.

\subsection{Value at risk}

We consider the chance constraint $\mathbb{P}[B(t ; \alpha) \geq 0] \geq \zeta$ for a given probability level $\zeta$. As mentioned above, technically we have to include the distribution of the constraint $B \geq 0$ depending on the uncertain recession strength $\alpha$ to calculate the appropriate probabilities. To overcome this difficulty, in the conspicuous consumption problem we can make use of Theorem 1 .

Corollary 1 With the assumptions of the original recession model given by (3) and (4) we can deduce for a random parameter $\alpha \in \mathcal{L}^{2}$ that

$$
\mathbb{P}[B(t ; \alpha) \geq 0] \geq \zeta \quad \Leftrightarrow \quad B\left(t ; \operatorname{VaR}_{\zeta}(\alpha)\right) \geq 0 .
$$

Proof The dynamics of the cash state $B$ are given by

$$
\dot{B}(t ; \alpha)=p(t) D_{\mathrm{R}}(A(t), p(t))-C+\delta B(t ; \alpha) .
$$

As

$$
B(t ; \alpha)=B(0 ; \alpha)+\int_{0}^{t} \dot{B}(s ; \alpha) \mathrm{d} s
$$

we obtain the variational differential equation 


$$
\begin{aligned}
B_{\alpha}(t ; \alpha)= & B_{\alpha}(0 ; \alpha)+\int_{0}^{t}\left[p_{\alpha}(s) D_{\mathrm{R}}(A(s), p(s))\right. \\
& \left.+p(s) \frac{\partial}{\partial \alpha} D_{\mathrm{R}}(A(s), p(s))+\delta B_{\alpha}(s ; \alpha)\right] \mathrm{d} s .
\end{aligned}
$$

From the results of Caulkins et al. (2011), Huschto et al. (2011) we assume both the price $p(\cdot)$ and the demand in the recession period $D_{\mathrm{R}}(A(\cdot), p(\cdot))$ to be monotonically decreasing in $\alpha$. Hence, as the initial cash state $B(0 ; \alpha)$ is independent of the recession strength, $\delta>0$, and the price $p$ and demand $D_{\mathrm{R}}$ are non-negative for all $t$, we conclude

$$
B_{\alpha}(t ; \alpha) \leq 0 \quad \forall t .
$$

Then we can apply Theorem 1 (for $c(B)=-B$ ) to deduce the result.

Hence, we obtain the optimal control problem

$$
\begin{array}{clc}
\max _{p(\cdot)} & \mathbb{E}\left[\Phi^{i}(\tau)\right] & \\
\text { s.t. } & \dot{A}(t)=\kappa(\gamma p(t)-A(t)), & t \in\left[0, t_{\mathrm{f}}\right], \\
& \dot{B}(t)=p(t) D_{\mathrm{R}}(A(t), p(t))-C+\delta B(t), & t \in[0, \tau], \\
& A(0)=A_{0}, \quad B(0)=B_{0}, & \\
& 0 \leq D_{\mathrm{R} / \mathrm{N}}(A(t), p(t)), & t \in\left[0, t_{\mathrm{f}}\right], \\
& p(t) \geq 0, & t \in\left[0, t_{\mathrm{f}}\right], \\
& 0 \leq B\left(t ; \operatorname{VaR}_{\zeta}(\alpha)\right), & t \in[0, \tau],
\end{array}
$$

for some given probability level $0<\zeta<1$ and the notations from before.

\subsection{Conditional value at risk}

Analogous to Sect. 3.3 we incorporate the CVaR constraint into our conspicuous consumption model. For fixed $t$, the probabilistic constraint becomes (remembering $-B(t ; \alpha) \leq 0)$

$$
\begin{aligned}
0 & \geq \operatorname{CVaR}_{\zeta}(-B(t ; \alpha)) \\
& =\min _{\vartheta \in \mathbb{R}}\left\{\vartheta+\frac{1}{1-\zeta} \mathbb{E}[\max \{0,-B(t ; \alpha)-\vartheta\}]\right\} .
\end{aligned}
$$

But as this constraint has to hold for all $t \in[0, \tau]$, i.e., during the overall possible recession, the control parameter $\vartheta$ in the minimization rule (29) becomes a control function $\vartheta(t)$. Thus, the resulting robust optimal control problems are

$$
\begin{array}{cll}
\max _{p(\cdot), \vartheta(\cdot)} & \mathbb{E}\left[\Phi^{i}(\tau)\right] & \\
\text { s.t. } & \dot{A}(t)=\kappa(\gamma p(t)-A(t)), & t \in\left[0, t_{\mathrm{f}}\right], \\
& \dot{B}(t)=p(t) D_{\mathrm{R}}(A(t), p(t))-C+\delta B(t), & t \in[0, \tau], \\
& A(0)=A_{0}, \quad B(0)=B_{0}, & \\
& 0 \leq D_{\mathrm{R} / \mathrm{N}}(A(t), p(t)), & t \in\left[0, t_{\mathrm{f}}\right], \\
& p(t) \geq 0, & t \in\left[0, t_{\mathrm{f}}\right], \\
& 0 \geq \vartheta(t)+\frac{1}{1-\zeta} \mathbb{E}[\max \{0,-B(t ; \alpha)-\vartheta(t)\}], & t \in[0, \tau],
\end{array}
$$


where the additional control function $\vartheta(\cdot)$ is necessary only during the recession and becomes redundant in a normal phase.

\section{Strengths and weaknesses of the robustification techniques}

\subsection{Coherent measures of risk and their economic impact}

In Rockafellar (2007) the important questions "What is risk?" and "How can we quantify it?" have been addressed. In that context two disparate ideas appear: The measures of deviation regard the amount of risk in a random variable as the degree of uncertainty in it, whereas the measures of the risk of loss quantify risk in terms of a surrogate for the overall costs that may occur.

The key in optimization under uncertainty thus is to quantify the risk of loss rather than the degree of uncertainty. If those quantities can be expressed by some functional $\mathcal{R}$, then the next important question presses into focus: What properties have to be fulfilled such that this functional is a good quantifier of risk? Artzner et al. (1999) provided an answer to these considerations by characterizing coherent measures of risk, Rockafellar (2007) extended the original idea. For this purpose they introduced axioms a risk measure should satisfy if it is "used to effectively regulate or manage risk" (Artzner et al. 1999). In terms of this methodology, a risk associated with a coherent measure becomes acceptable if the corresponding constraints are satisfied. Otherwise the risk might be unacceptable if, e.g., the measure underestimates the consequences of failure. Still, the concept allows the original constraints to be violated. The important notion here is the extent of that violation.

The traditional approaches in optimization under uncertainty, i.e., guessing the future, worst-case analysis, and relying on expectations are basically coherent measures of risk in the sense of Artzner et al. (1999). Nevertheless, they inherit many disadvantages. Worst-case approaches take into account every possible outcome of the uncertain parameters, no matter how unlikely it may be. While this characteristic of the worst-case approaches becomes crucial in applications like safeguarding chemical processes or power plants, in economic situations it is often too conservative. In many such circumstances decision makers take into account a certain amount of risk of failure to achieve greater gains. In contrast to the worstcase approaches expectation-based ideas provide acceptable risks even if desirable outcomes merely compensate the undesirable ones. Hence, they are often too optimistic to be applied in questions of economics. Both here proposed approaches of robust worst-case optimal control, i.e., the linearization and sigmapoint methods, allow the investigation of desired confidence levels $\omega$ if the variance $\Sigma$ is known. Otherwise combinations of both notions have to be considered. Therein, the sigmapoint approach is advantageous from the economic point of view as it allows deeper economic insight in the behavior of the solution.

To overcome the general difficulties of worst-case and expectation-based ideas, especially in the field of finance, the VaR attracted much attention. Unfortunately, despite its broad usage, it is generally not a coherent measure of risk (Artzner et al. 
1999; Rockafellar 2007). Speaking in terms of portfolio optimization, the VaR does not satisfy the diversification principle (Artzner et al. 1999). Moreover, it tends towards optimistic estimations of uncertain situations as it does not provide a grasp on the seriousness of constraint violations (Rockafellar 2007). These properties are a severe disadvantage in risk management or in an economic situation where the firm has the possibility to borrow money at the market and the corresponding interest rates increase with the amount of needed cash. In our considered conspicuous consumption model with malfunctioning capital markets, however, the extent of violating a constraint, namely the bankruptcy constraint $B \geq 0$, is less important, as the firm has to face bankruptcy in any case where $B$ becomes negative. Thus, the negative connotation of the $\mathrm{VaR}$ is unjustified in our special economic case.

As mentioned before, the $\mathrm{CVaR}$ provides a more cautious approach to safeguarding than the incorporation of pure chance constraints by the VaR because it rates constraint violations caused by decisions. In addition, it has been proven under various assumptions that the $\mathrm{CVaR}$ describes a coherent measure of risk (Acerbi and Tasche 2002; Rockafellar and Uryasev 2002), which constitutes it to be a reliable quantifier of risk. However, in our special economic situation the classification of constraint violations using the $\mathrm{CVaR}$ is a subordinate issue, which can cause the resulting pricing strategies to be very risk-averse or even too conservative.

\subsection{Numerical expenses}

Besides the theoretical and economical aspects of using the presented robustification techniques, there are as well broad differences from the numerical point of view.

The general formulation of a robust worst-case constraint (7) leads to a semiinfinite optimal control problem that is very hard to solve numerically. Therefore, an approximation of (7) by either the linearization or the sigmapoint approach is necessary. The resulting problems (9) and (12) can be efficiently solved by existing methods like, e.g., Bock's direct multiple shooting approach (Bock and Plitt 1984). For highly nonlinear problems linearization idea can cause approximation errors, whereas the robustness of solutions obtained by it cannot be guaranteed. As a remedy higher order approximation schemes may become useful, compare the method proposed by Heine et al. (2006). Another possibility (Diehl et al. 2006, 2008 ) is to replace the inner minimization term by its sufficient optimality condition. All of the listed ideas result in a far more difficult problem as additional equations have to be considered. Consequently, the computational effort increases considerably. Within the sigmapoint approach, however, the computational complexity is extended by additional path constraints on the state variable depending on the propagated sigmapoints (24a).

In general the $\mathrm{VaR}$ is difficult to work with numerically, e.g., if loss distributions feature "fat tails" or jumps; compare Uryasev (2002). In our context and reasoned by the considerations in Sect. 4.3 we only need the distribution of $\alpha$ to calculate its $\mathrm{VaR}$ for a given confidence level $\zeta$. Thus, the incorporation of the VaR constraint in the conspicuous consumption model can be done very efficiently as only one additional state variable and path constraint are needed. 
By contrast, implementing the $\mathrm{CVaR}$ is far more complex. The minimization rule (29) induces that besides the additional control variable $\vartheta(\cdot)$ the calculation of the expectation within the formula is needed. This can be achieved by introducing auxiliary cash state variables depending on values the random variable $\alpha$ attains with a corresponding probability. Thus, the computational effort increases with the number of those auxiliary variable.

To illustrate the differences in the expenses of solving the multi-stage optimal control problems resulting from the different robustification methods, Table 1 presents the overall dimensions of the Nonlinear Programs obtained by transforming those problems with Bock's direct multiple shooting approach. It discretizes the space of admissible control functions and the constraints. The ODEs of the system's dynamics are solved via decoupled integration on a multiple shooting grid. Therefore, artificial intermediate variables are introduced as starting values of the integration, continuity of the states is assured by the inclusion of matching conditions. A detailed introduction to this method is given in Bock and Plitt (1984), Leineweber et al. (2003).

The resulting structured nonlinear program (NLP) is then solved by a specially tailored sequential quadratic programming (SQP) algorithm (Leineweber et al. 2003). Therein, the principle of internal numerical differentiation (IND) is used to derive the sensitivities of the ODE solution (Albersmeyer and Bock 2008) and a condensing algorithm (Bock and Plitt 1984; Leineweber et al. 2003) to obtain small dense quadratic programs $(\mathrm{QP})$ that are solved within each SQP iteration to progress towards the NLP solution.

Within Table 1 we can see that the smallest resulting problem we obtain when using the VaR approach. As more additional state variables and/or constraints are needed for both robust worst-case approaches, those problems are slightly larger, whereas the CVaR is the largest one. This is mainly caused by the second control function required to implement the constraint. These variations are reflected in the CPU time behavior as well, compare Table 2. While the differences in the number of state functions of the linearization/sigmapoint/VaR approach do not influence the average runtime (per SQP iteration) and its distribution among the parts of the solving procedure much, the additional control function of the CVaR approach does. The runtime of that robustification method is noticeably higher, with the effort in solving the resulting QPs becoming more prominent.

\section{Numerical results}

The numerical results presented in the following are based on the parameters [compare Caulkins et al. (2010, 2011)]

$$
\begin{array}{ll}
\kappa=2.0, & \gamma=5.0, \quad C=7.5, \quad \delta=0.05, \\
m=3.0, \quad \beta=0.5, \quad r=0.1, \quad \lambda=0.5,
\end{array}
$$

and the assumptions (Huschto et al. 2011)

$$
\tau_{n}=20 \text { (years) } \quad t_{\mathrm{f}}=21 \text { (years). }
$$


Table 1 Dimensions of the Nonlinear Programming (NLP) problems resulting from the conspicuous consumption problem with the presented robustification techniques (linearization and sigmapoint approach, $\mathrm{VaR}$ and $\mathrm{CVaR}$ ) and choosing between the objective functions (22a) determined by $\alpha=\bar{\alpha}$ and (22b) determined by $\alpha=0$

\begin{tabular}{lccrr}
\hline & Linearization (L) & Sigmapoint (S) & VaR (V) & CVaR (C) \\
\hline Objective function $\Phi^{1}$ & $(22 \mathrm{a})$ & & & \\
\# discr. points & 1,840 & 1,840 & 1,840 & 1,840 \\
\# variables & 12,957 & 11,195 & 9,316 & 18,632 \\
\# eq. constr. & 11,112 & 9,351 & 7,473 & 14,946 \\
\# ineq. constr. & 27,754 & 27,910 & 18,752 & 37,384 \\
Objective function $\Phi^{2}$ & $(22 \mathrm{~b})$ & & & 1,840 \\
\# discr. points & 1,840 & 1,840 & 1,840 & 20,511 \\
\# variables & 16,676 & 13,074 & 9,316 & 16,824 \\
\# eq. constr. & 14,829 & 11,229 & 7,473 & 41,142 \\
\# ineq. constr. & 35,192 & 31,668 & 18,752 & \\
\hline
\end{tabular}

The smallest problem we obtain for the VaR approach, the largest one for the CVaR. This is caused by the necessity of auxiliary cash state variables and, mainly, an additional control function

Table 2 Exemplary computation times in h:min:s for solving the NLP problems of Table 1

\begin{tabular}{|c|c|c|c|c|c|c|c|c|}
\hline & \multicolumn{2}{|c|}{ Linearization (L) } & \multicolumn{2}{|c|}{ Sigmapoint (S) } & \multicolumn{2}{|l|}{$\operatorname{VaR}(\mathrm{V})$} & \multicolumn{2}{|l|}{$\mathrm{CVaR}(\mathrm{C})$} \\
\hline \multicolumn{9}{|c|}{ Objective function $\Phi^{1}$ (22a) } \\
\hline IND & $1: 19$ & $(1.0 \%)$ & 24 & $(0.3 \%)$ & 11 & $(0.2 \%)$ & $1: 00$ & $(0.3 \%)$ \\
\hline state int. & 15 & $(0.2 \%)$ & 5 & $(0.1 \%)$ & 3 & $(0.0 \%)$ & 8 & $(0.0 \%)$ \\
\hline condens. & $1: 59: 04$ & $(87.3 \%)$ & $2: 06: 42$ & $(84.6 \%)$ & $1: 34: 02$ & $(86.9 \%)$ & $4: 24: 36$ & $(67.9 \%)$ \\
\hline solv. QP & $15: 46$ & $(11.5 \%)$ & $22: 27$ & $(15.0 \%)$ & $13: 59$ & $(12.9 \%)$ & $2: 03: 56$ & $(31.8 \%)$ \\
\hline \multirow[t]{3}{*}{ rest } & 3 & $(0.0 \%)$ & 2 & $(0.0 \%)$ & 2 & $(0.0 \%)$ & 4 & $(0.0 \%)$ \\
\hline & \multicolumn{2}{|l|}{$2: 16: 27$} & \multicolumn{2}{|l|}{$2: 29: 40$} & \multicolumn{2}{|l|}{ 1:48:17 } & \multicolumn{2}{|l|}{$6: 29: 44$} \\
\hline & \multicolumn{2}{|l|}{ (39 SQP) } & \multicolumn{2}{|l|}{ (43 SQP) } & \multicolumn{2}{|l|}{ (34 SQP) } & \multicolumn{2}{|l|}{ (62 SQP) } \\
\hline \multicolumn{9}{|c|}{ Objective function $\Phi^{2}(22 b)$} \\
\hline IND & $2: 26$ & $(1.5 \%)$ & 26 & $(0.4 \%)$ & 13 & $(0.1 \%)$ & 52 & $(0.3 \%)$ \\
\hline state int. & 21 & $(0.2 \%)$ & 5 & $(0.1 \%)$ & 4 & $(0.1 \%)$ & 6 & $(0.0 \%)$ \\
\hline condens. & $2: 17: 26$ & $(86.6 \%)$ & $1: 44: 53$ & $(86.2 \%)$ & $2: 20: 47$ & $(86.5 \%)$ & $3: 29: 15$ & $(70.0 \%)$ \\
\hline solv. QP & $18: 21$ & $(11.6 \%)$ & $16: 12$ & $(13.3 \%)$ & $21: 41$ & $(13.3 \%)$ & $1: 28: 49$ & $(29.7 \%)$ \\
\hline \multirow[t]{3}{*}{ rest } & 4 & $(0.1 \%)$ & 2 & $(0.0 \%)$ & 2 & $(0.0 \%)$ & 4 & $(0.0 \%)$ \\
\hline & \multicolumn{2}{|l|}{$2: 38: 38$} & \multicolumn{2}{|l|}{$2: 01: 38$} & \multicolumn{2}{|l|}{$2: 42: 47$} & \multicolumn{2}{|l|}{$4: 59: 06$} \\
\hline & \multicolumn{2}{|l|}{ (41 SQP) } & \multicolumn{2}{|l|}{ (35 SQP) } & \multicolumn{2}{|l|}{ (42 SQP) } & \multicolumn{2}{|l|}{ (42 SQP) } \\
\hline
\end{tabular}

Note that solving the problem with CVaR constraint is most expensive, due to the additional control function. The remaining approaches need comparable computation times 
In addition, we choose the initial reputation and cash to be

$$
\begin{aligned}
& A_{0}^{1}=20.0, \quad B_{0}^{1}=10.0, \\
& A_{0}^{2}=40.0, \quad B_{0}^{2}=50.0,
\end{aligned}
$$

each of which corresponds to economic starting points, where the firm can cope with an intermediate $\left(A_{0}^{1}, B_{0}^{1}\right)$ or severe $\left(A_{0}^{2}, B_{0}^{2}\right)$ recession for a certain time period, but which does not hold enough capital reserves to survive a continuing recession (Caulkins et al. 2011; Huschto et al. 2011). Finally, the random variable $\alpha$ for the linearization and the sigmapoint approaches is characterized by its mean value

$$
\bar{\alpha}=0.836
$$

and varying values of variance $\Sigma$. While analyzing the $\mathrm{VaR}$ and $\mathrm{CVaR}$, we assume that we know a certain distribution of the random variable, e.g., by historical data. Thus, we define $\alpha$ of these approaches through

$$
\begin{aligned}
& \mathbb{P}[\alpha=0.5]=0.1 \\
& \mathbb{P}[\alpha=0.7]=0.3 \\
& \mathbb{P}[\alpha=0.836]=0.4 \\
& \mathbb{P}[\alpha=0.9]=0.15 \\
& \mathbb{P}[\alpha=1.25]=0.05 .
\end{aligned}
$$

\subsection{Linearization and sigmapoint approaches}

Both methods to approximate the robust worst-case formulation (7) depend only on the variance $\Sigma$ if we fix the confidence level $\omega=1$, i.e., considering a combination of these two notions as we assume the exact variance of the random recession strength to be unknown. Figures 2 and 3 depict the optimal price paths in the recession period $[0, \tau]$ of problems $(\mathrm{L})$ and $(\mathrm{S})$, respectively, when the objective function is $\mathbb{E}\left[\Phi^{1}(\tau)\right]$ and initial values $A_{0}^{1}, B_{0}^{1}$ are used.

We notice that both approaches yield equal optimal pricing strategies and objective values (compare Table 3). For very low variances the prices during the recession phase do not have to be reduced, as the objective function already includes some caution towards the realization of $\alpha$ and the initial cash $B_{0}$ is enough to keep the cash state positive during the complete recession even if the worst possible outcome of $\alpha$ based on $\Sigma$ takes place. For higher variances the decision maker has to decrease prices to survive the recession.

In addition, Table 3 shows how much of the overall gains is lost, if decision makers have to reduce prices according to an uncertain recession strength with mean $\bar{\alpha}$ and variance $\Sigma$. Note that the actual profit is obtained during the normal economic stage, but is strongly depending on the reputation level the firm can keep during the crisis.

In the phase diagrams of Figs. 2 and 3 the implications of decreasing prices can be seen: consistent with its dynamics the firm's brand image is damaged as well. The great economic advantage of the sigmapoint approach here is that due to the additional cash state variables needed to implement the modified constraints based 
Robust linearized constraint with $\bar{\alpha}$ in objective and initial values $A_{0}^{1}, B_{0}^{1}$

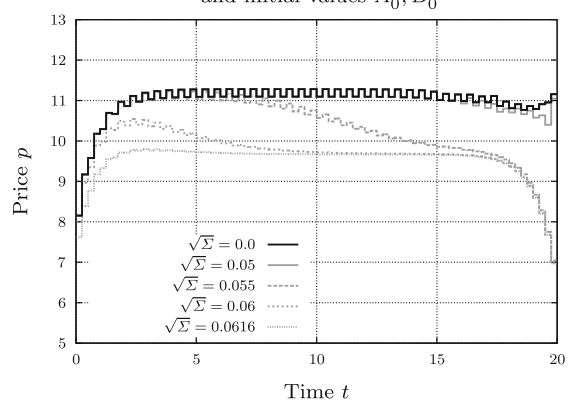

Robust linearized constraint with $\bar{\alpha}$ in objective and initial values $A_{0}^{1}, B_{0}^{1}$

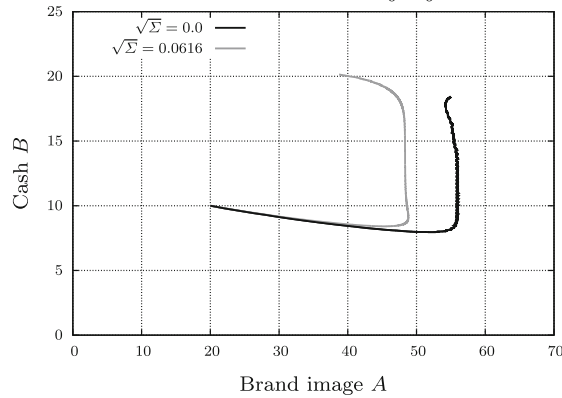

Fig. 2 Robust price paths of the recession phase (left) and exemplary phase diagram (right) for problem (L) with objective function $\Phi^{1}$ obtained using the linearization approach (23). The price paths depend on the variance $\Sigma$ of the random recession strength if we fix the confidence level $\omega=1$. Then higher variances require decision makers to decrease prices appropriately

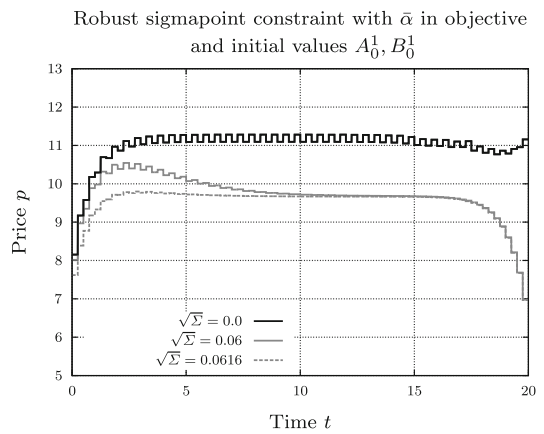

Robust sigmapoint constraint with $\bar{\alpha}$ in objective, $\sqrt{\Sigma}=0.0616$,

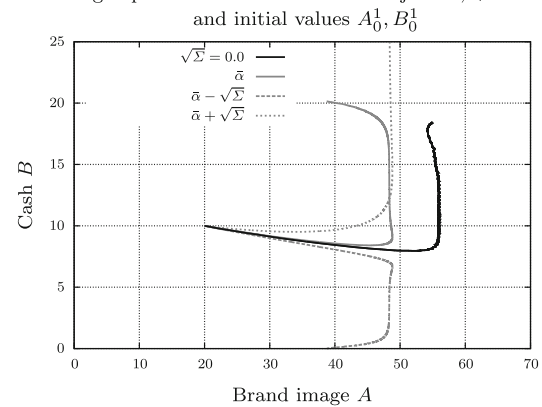

Fig. 3 Robust price paths of the recession phase (left) and exemplary phase diagram (right) for problem (S) with objective function $\Phi^{1}$ obtained using the sigmapoint approach (23). The phase diagram depicts the connection of brand image state and the cash states depending on the sigmapoints $\alpha_{i}, i=0,1,2$

on the sigmapoints (especially for $\alpha_{2}=\bar{\alpha}+\sqrt{\Sigma}$ ), we can see the actual reason of reducing prices. It is caused by the decision maker's optimal strategy to balance prices in a way that the firm operates into a "zero cash-situation" at time $t=20$ (years) when the economic crisis will finally be over (due to our assumption of $\tau_{n}=20$ ). Naturally, in an ever-lasting recession the firm finally has to face bankruptcy, if its initial reputation and cash stock are not sufficiently high. ${ }^{1}$

However, the decision maker's optimal strategy is based on another important principle. Prices have to be kept as high as possible as long as possible to preserve the reputation of the product, as this will guarantee the firms success once the crisis is over. Therefore, in the beginning of the recession the optimal strategy is charging

\footnotetext{
${ }^{1}$ For a detailed description of the pricing strategies depending on the economic stages, the initial cash and reputation, time delays, and the three major types of recession, compare Caulkins et al. (2011), Huschto et al. (2011).
} 
Table 3 Optimal objective values $\Phi^{*}$ for the robust conspicuous consumption problems (L)-(C)

\begin{tabular}{|c|c|c|c|c|c|c|c|}
\hline \multicolumn{2}{|c|}{ Linearization (L) } & \multicolumn{2}{|c|}{ Sigmapoint (S) } & \multicolumn{2}{|c|}{$\mathrm{VaR}(\mathrm{V})$} & \multicolumn{2}{|c|}{ CVaR (C) } \\
\hline$\sqrt{\Sigma}$ & $\Phi^{*}$ & $\sqrt{\Sigma}$ & $\Phi^{*}$ & $\zeta$ & $\Phi^{*}$ & $\zeta$ & $\Phi^{*}$ \\
\hline \multicolumn{8}{|c|}{ Objective function $\Phi^{1}(22 \mathrm{a})$ and $\left(A_{0}^{1}, B_{0}^{1}\right)$} \\
\hline 0.0 & 88.5342 & 0.0 & 88.5342 & 0.0 & 88.5344 & 0.0 & 88.5344 \\
\hline 0.06 & 88.5055 & 0.06 & 88.5055 & 0.9 & 88.5344 & 0.4 & 88.5343 \\
\hline \multirow[t]{3}{*}{0.0616} & 88.4009 & 0.0616 & 88.4009 & 0.92 & 88.5343 & 0.45 & 88.5338 \\
\hline & & & & 0.93 & 88.5338 & & \\
\hline & & & & 0.94 & 88.5099 & & \\
\hline \multicolumn{8}{|c|}{ Objective function $\Phi^{2}(22 \mathrm{~b})$ and $\left(A_{0}^{1}, B_{0}^{1}\right)$} \\
\hline 0.0 & 106.8768 & 0.0 & 106.8768 & 0.0 & 107.6456 & 0.0 & 107.2890 \\
\hline 0.02 & 106.3943 & 0.02 & 106.3943 & 0.4 & 107.6321 & 0.3 & 106.2436 \\
\hline 0.04 & 105.3583 & 0.04 & 105.3583 & 0.8 & 106.8768 & 0.4 & 104.3689 \\
\hline 0.06 & 102.4368 & 0.06 & 102.4368 & 0.9 & 105.1500 & 0.45 & 103.6880 \\
\hline 0.0616 & 101.4003 & 0.0616 & 101.4003 & 0.94 & 102.5151 & & \\
\hline \multicolumn{8}{|c|}{ Objective function $\Phi^{2}(22 b)$ and $\left(A_{0}^{2}, B_{0}^{2}\right)$} \\
\hline 0.0 & 146.5020 & 0.0 & 146.5020 & 0.0 & 146.5021 & 0.0 & 146.5019 \\
\hline 0.06 & 146.5006 & 0.06 & 146.5006 & 0.4 & 146.5021 & 0.8 & 146.4537 \\
\hline 0.2 & 146.4839 & 0.2 & 146.4839 & 0.9 & 146.5015 & 0.9 & 145.7950 \\
\hline 0.3 & 145.5607 & 0.3 & 145.5607 & 0.96 & 146.4734 & 0.92 & 145.7755 \\
\hline 0.4 & 143.0664 & 0.4 & 143.0664 & 0.99 & 144.7815 & 0.95 & 142.4378 \\
\hline 0.414 & 142.4379 & 0.414 & 142.4379 & 1.0 & 142.4379 & & \\
\hline
\end{tabular}

It is shown how much is lost if we regard the different approaches with varying values of variance $\Sigma$ $((\mathrm{L})$ and $(\mathrm{S}))$ or $\zeta$-level $((\mathrm{V})$ and $(\mathrm{C}))$ in comparison with the nominal solution (i.e., $\Sigma=0$ or $\zeta=0$ ). Note that the major part of this objective value is obtained during the normal economic phase depending on the performance during the recession. We can see that both methods of robust worst-case approximation give equal results. Further on, the relation between the $\zeta$-levels in $(\mathrm{V}) /(\mathrm{C})$ and the confidence levels in (L)/ (S) (included indirectly in the variances) is observable, as well as the differences in the cautiousness of (V) and (C). Note that the gaps in the nominal objective values (regarding $\Sigma=0.0 / \zeta=0.0$ ) are directly caused by the formulations of the corresponding constraint

the optimal, i.e., highest possible price for the chosen objective function assuming there is no chance of a stronger crisis. Only when the recession persists longer, prices eventually have to be reduced according to the worst possible realization of $\alpha$ determined by the variance $\Sigma$ and the firm's incentive to keep cash until $\tau_{n}$. By this strategy the brand image remains at a high level in the first period of the recession when it is very probable that $\tau$ is reached soon. In that situation the gains of the normal economic stage are higher as if the decision maker set a constant price during the longest possible duration $\tau_{n}$ of the recession. The same behavior can often be observed at the very end of the longest possible recession: Rather than fixing the price at some constant level $\tilde{p}$, it is more profitable to reduce prices considerably at the last possible instance when this measure is successful and concurrently being able to set a (slightly) higher price $p>\tilde{p}$ in the period before. The general effect can be noticed in Figs. 2 and 3 but more obviously in Fig. 4, 
which shows the optimal price paths of problem (L) with the objective function $\mathbb{E}\left[\Phi^{2}(\tau)\right]$ and both sets of initial values. Clearly, it is more apparent for smaller variances.

Moreover, in Fig. 4 the connection between the variance and the reduction of prices is observable more directly as the objective of the firm depends on the norecession situation. In addition, the right plot shows optimal prices if the firm starts with a higher initial reputation and capital stock. Then it can even cope with situations where the variance of the random recession strength is assumed to be relatively large, including the (worst possible) realization of a severe recession characterized by $\alpha=1.25$. This results because for the set of large initial values $\left(A_{0}^{2}, B_{0}^{2}\right)$ prices can be decreased further than for the set of small initial values. For the latter set, we cannot calculate solutions corresponding to large variances of $\alpha$ or even the worst case, as this solution is infeasible.

\subsection{Value at risk}

To calculate the VaR of the random recession strength we use the definition (32) of $\alpha$. Therefore, the mean of the random variable defined by that distribution varies a little from the value $\bar{\alpha}$ we have used in the last two sections. Nevertheless, for reasons of comparison, we still implement the first alternative of the objective function $\Phi^{1}$ with $\bar{\alpha}$. The actual quantiles of the recession strength corresponding to a given probability level $\zeta$ can be obtained by linear interpolation of the distribution given in (32).

Figures 5, 6, 7 depict solutions of Problem (V). As already noticed in the previous subsection for the robust worst-case approaches, when considering the objective function $\Phi^{1}$ including a pre-assumption of an intermediate recession strength, prices have to be reduced only for relatively large probability levels $\zeta$. Therefore, economic challenges favor the second choice of objective function $\Phi^{2}$ based on a no-recession scenario. Then the connection between the desired confidence level $\zeta$ and the price reductions becomes more apparent.

From comparing the optimal objective values of the VaR and the robust worstcase approaches in Table 3, we see a certain correspondence between the probability levels $\zeta$ of $\mathrm{VaR}$ and the confidence level/variance-combination within the robust worst-case formulations, even as they are based on very different assumptions on the random variable $\alpha$. In contrast to the linearization and sigmapoint idea, the nominal solution of the VaR approach with objective function $\Phi^{2}$ is obtained by the constraint $\mathbb{P}[B(t ; \alpha) \geq 0] \geq 0$, i.e., by a constraint that considers a no-recession scenario. Thus, the corresponding objective value is higher than for the robust worst-case formulations.

Like in the sigmapoint approach, the additional state variable $B\left(t, \operatorname{VaR}_{\zeta}(\alpha)\right)$ needed to implement the chance constraint allows for more economic insight, as we can see how the firm's cash evolves into zero when the crisis lasts for the worst possible duration $\tau_{n}$. Furthermore, in the phase diagrams of Figs. 6 and 7 (observe the trajectories corresponding to the probability level $\zeta=0.4$ ) we can see how the initial conditions impinge on a long persisting recession: While it is not possible for 
Robust linearized constraint with $\alpha=0$ in objective and initial values $A_{0}^{1}, B_{0}^{1}$

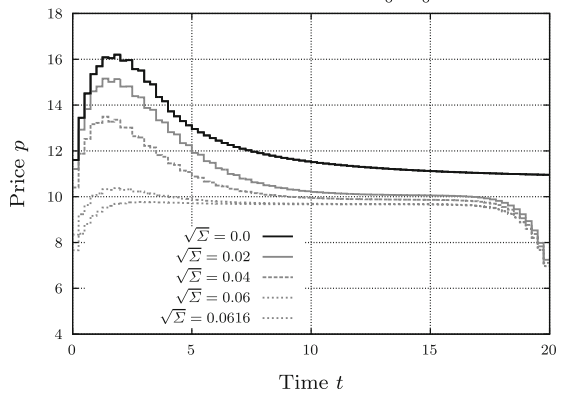

Robust linearized constraint with $\alpha=0$ in objective and initial values $A_{0}^{2}, B_{0}^{2}$

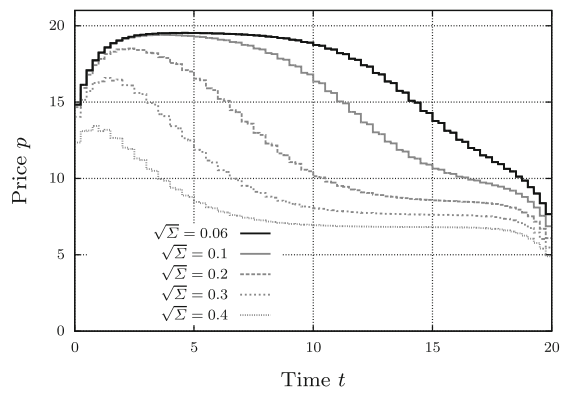

Fig. 4 Robust price paths of the recession phase as in Fig. 2, but for the objective function $\Phi^{2}$ and initial values $A_{0}^{1}, B_{0}^{1}$ (left) and $A_{0}^{2}, B_{0}^{2}(r i g h t)$. With higher initial values the firm can cope with more serious situations
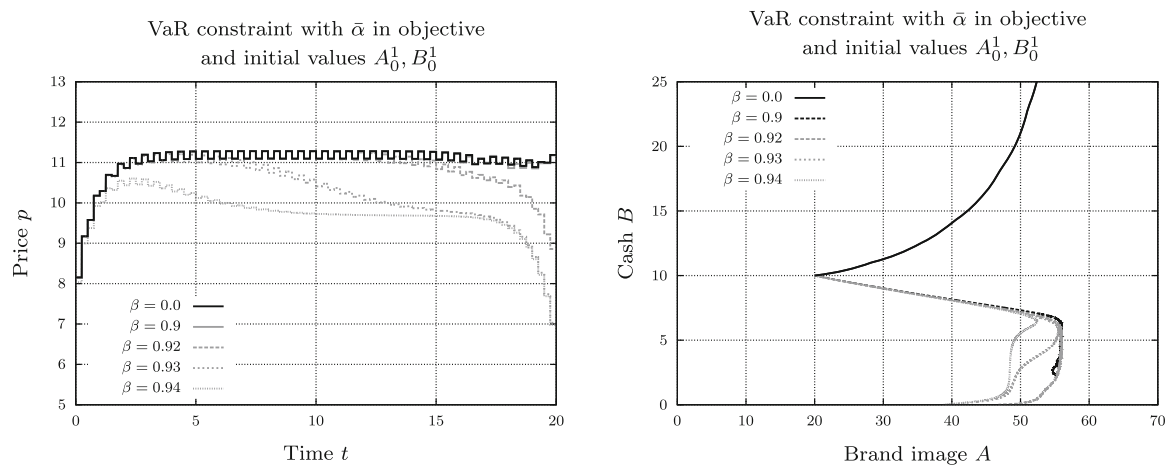

Fig. 5 Robust price paths of the recession phase (left) and exemplary phase diagram (right) for problem (V) with objective function $\Phi^{1}$ obtained using the probability constraint as in Corollary 1 . In the phase diagram the brand image is plotted against the cash state variable $B\left(t ; \operatorname{VaR}_{\zeta}(\alpha)\right)$ corresponding to the desired confidence level $\zeta$
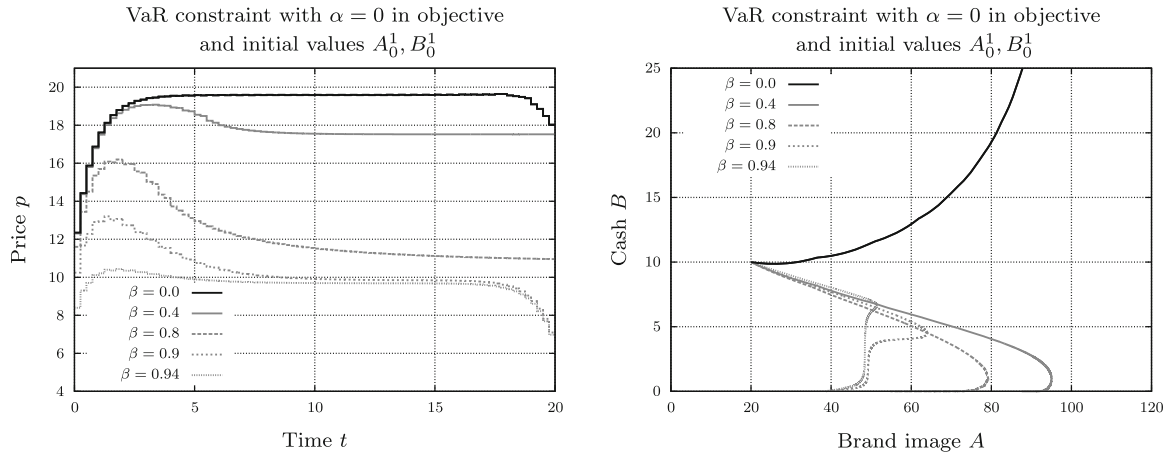

Fig. 6 Robust price paths of the recession phase and phase diagram as in Figure 5, but for the objective function $\Phi^{2}$ and initial values $A_{0}^{1}, B_{0}^{1}$ 
the firm to survive a very long $\left(\tau>20=\tau_{n}\right)$ recession with initial conditions $\left(A_{0}, B_{0}\right)=(20.0,10.0)$ for the corresponding recession strength $(B$ evolves towards zero), this is the case if the initial conditions are $\left(A_{0}, B_{0}\right)=(40.0,50.0)(B$ evolves to infinity).

In general we can observe that applying the VaR as robustification measure leads to very reasonable pricing strategies depending on the probability levels $\zeta$ and, therefore, the VaR of the uncertain recession strength $\alpha$ based on its definition (32). Due to the fact that a rating of violations of the constraint $\operatorname{VaR}_{\zeta}(B(t ; \alpha)) \geq 0$ plays a subordinate role in the conspicuous consumption model, the results do not suffer from the VaR not being a coherent measure of risk.

\subsection{Conditional value at risk}

Again, we use $\alpha$ as defined in (32), but include $\bar{\alpha}$ in the first objective function $\Phi^{1}$. Furthermore, the expectation operator within the CVaR constraint (29) turns into a summation due to the definition of $\alpha$.

The solutions to Problem (C) for both variants of the objective function and both sets of initial values can be seen in Figs. 8 and 9. The price paths behave qualitatively equal as in the aforementioned approaches, apart from that prices obtained with a $\mathrm{CVaR}$ constraint are more cautious than prices obtained with, e.g., a $\mathrm{VaR}$ constraint (compare the objective values and corresponding $\zeta$-levels in Table 3 as well). It means that for a given confidence level $\zeta$ the corresponding prices $p_{\mathrm{CVaR}}(\cdot)$ obtained with a $\mathrm{CVaR}$ constraint are lower than the prices $p_{\mathrm{VaR}}(\cdot)$ obtained with one of the other approaches, e.g., the VaR. Compare, for instance, the first plots in Figs. 7 and 9.

Moreover, in Table 3 it can be noted that the nominal value of the CVaR approach is based on the $\mathrm{CVaR}$ constraint corresponding to the expectation value $\mathbb{E}[B(t ; \alpha)]$, compare Theorem 2 . This is again different from the robust methods and the VaR, where the nominal solution is based on a constraint with $\bar{\alpha}$ and $\alpha=0$, respectively, compare Remark 1 . The cautiousness of this method is reflected in the optimal objective values of corresponding $\zeta$-levels as well.

With linearized robustification, sigmapoints, and the VaR we obtain a cash state $B$ that corresponds directly to the robustified constraint. Hence, we can analyze the behavior of the constraint in a phase diagram of reputation $A$ and cash $B$. In the $\mathrm{CVaR}$ approach this is not the case, as the constraint is realized via the minimization rule (29). Therefore, in both Figs. 8 and 9 we depict cash state trajectories during the recession phase $\left[0, \tau_{n}\right]$ for a specific realization of the random variable $\alpha$, i.e., $\alpha=0.9$ which occurs with a probability of 15 percent. One notices (e.g., from Fig. 8) that the cash state $B(t, \alpha=0.9)$ corresponding to a recession with strength $\alpha=0.9$ can drop below zero and still the desired confidence level (of, e.g., $\zeta=0.45)$ is reached. If the confidence level is increased, then to fulfill this level prices have to be adjusted in a way such that eventually the cash state for $\alpha=0.9$ remains positive for all possible durations of the recession and only the cash states corresponding to the severe recession may become negative. 

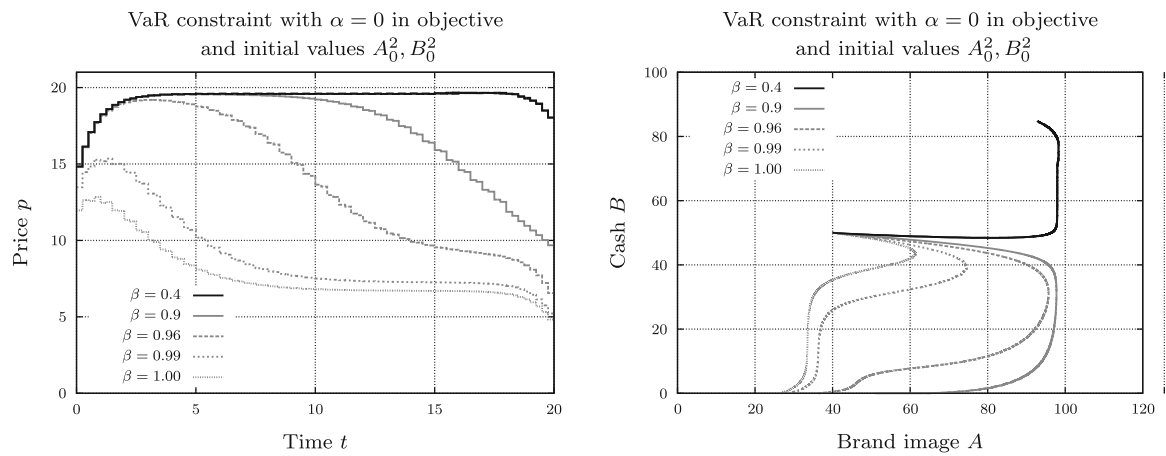

Fig. 7 Robust price paths of the recession phase and phase diagram as in Fig. 5, but for the objective function $\Phi^{2}$ and initial values $A_{0}^{2}, B_{0}^{2}$

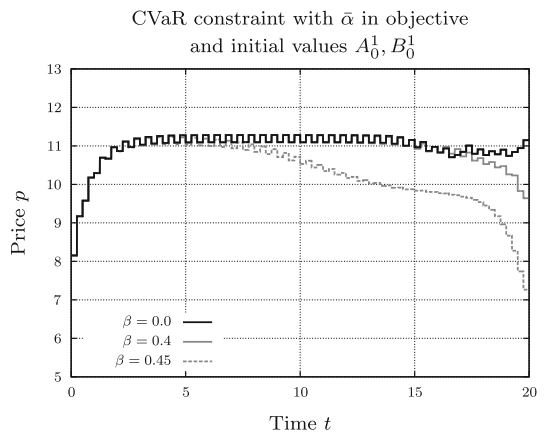

Evolution of cash state $B(t, \alpha=0.9)$ for CVaR constraint with

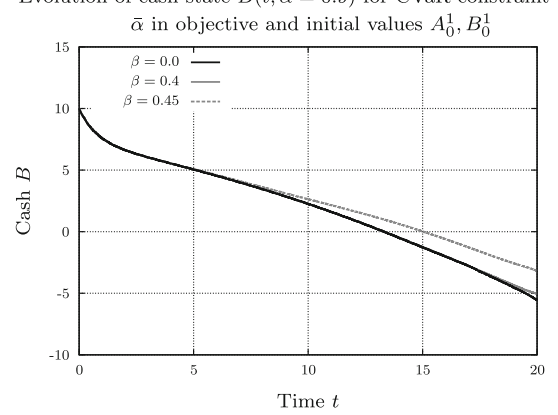

Fig. 8 Robust price paths of the recession phase (left) and exemplary cash state trajectory $B$ during this phase (right) for problem (C) with objective function $\Phi^{1}$. The cash trajectory is depicted for a intermediate recession of strength $\alpha=0.9$
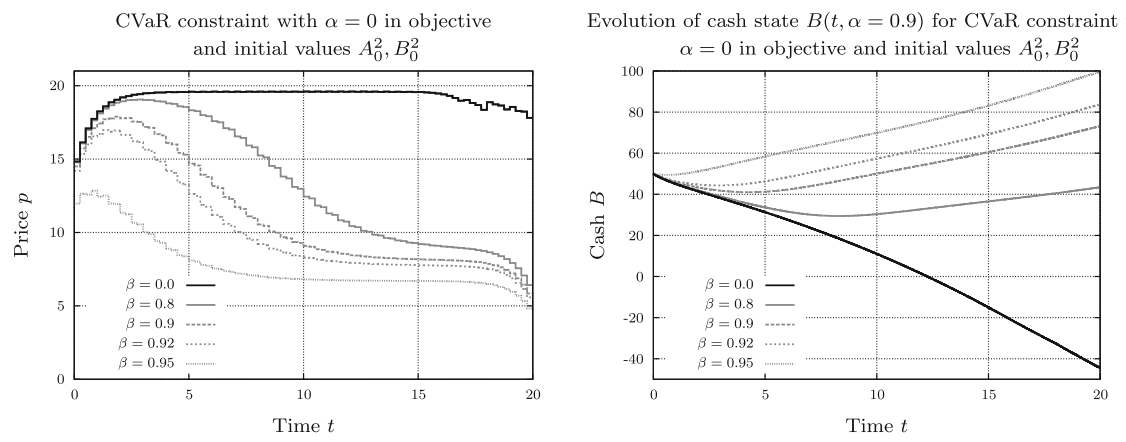

Fig. 9 Robust price paths of the recession phase and phase diagram as in Fig. 8, and for the objective function $\Phi^{2}$ and initial values $A_{0}^{2}, B_{0}^{2}$ 
Furthermore, the approach tends to be a bit too conservative in the context of the conspicuous consumption problem caused by the classification of constraint violations in the CVaR approach due to the minimization formula (29). Hence, the $\mathrm{CVaR}$ is a very risk-averse version of safeguarding.

\section{Summary and outlook}

In the context of the considered conspicuous consumption model with uncertain recession strength we implemented and compared different esteemed approaches of optimization under uncertainties to the special case of optimal control in economics.

Based on the desired confidence level decision makers have to adjust prices to survive the recession. In general, price reductions are directly connected to how conservative the decision should be. But this reduction is optimally conducted adaptively depending on the (uncertain) duration of the recession rather than to some fixed price that holds over the complete crisis. This behavior, recalling the feedback character of stochastic control, is caused by the firm's incentive to retain a high reputation in the time when the recession will end most probably.

For obtaining these results, we discussed a linearization and the sigmapoint approach to efficiently reformulate robust worst-case optimal control problems into numerically solvable ones. While both approaches lead to similar computationally complex problems, the economic insight provided by the sigmapoint approach is very beneficial. However, as often in the context of worst-case optimization, both approaches may lead to infeasible solutions. In our consumption problem this is the case if we consider sets of low initial reputation and cash and large variances in the uncertain recession strength.

While worst-case approximations are often too conservative to apply in economic situations, the ideas of $\mathrm{VaR}$ and $\mathrm{CVaR}$ offer economic decision makers the opportunity to balance their decisions in a way that they can risk negative outcomes (bankruptcy) for the sake of profit-making. We included both concepts into the optimal control problem using special reformulations of the constraints $(\mathrm{VaR})$ or the original definitions $(\mathrm{CVaR})$. Thereby, the $\mathrm{VaR}$ approach can be implemented with a complexity that is slightly lower than for the robust worst-case approaches, while the $\mathrm{CVaR}$ is computationally expensive.

In the context of coherent measures of risk, the VaR is often estimated negatively, because it is no such measure, while the CVaR is, as it classifies the strength of negative outcomes. However, in the conspicuous consumption model this principle is less significant as in other, i.e., financial applications: If the firm runs out of cash and has no possibility to lend money at the market, it has to face bankruptcy no matter how much the constraint $B \geq 0$ is violated. Hence, the VaR approach leads to reliable pricing strategies here. The $\mathrm{CVaR}$ idea instead is a very risk-averse version of safeguarding in that context, may be even a bit too conservative. Nevertheless, both the $\mathrm{VaR}$ and $\mathrm{CVaR}$ approaches can yield infeasible solutions as well if the initial values are too low for the desired confidence level to be fulfilled. 
An interesting extension for all ideas in robust optimization towards a more realistic behavior of the proposed model is to consider time-dependent uncertain parameters. Up to now, the (fixed and constant) realization of the parameter is known directly after the start, but in many applications it may change over time. In that case all states and controls become random processes which require the development of entirely new methods in robust optimization.

\section{References}

Acerbi C (2002) Spectral measures of risk: a coherent representation of subjective risk aversion. J Bank Finance 26:1505-1518

Acerbi C, Tasche D (2002) On the coherence of expected shortfall. J Bank Finance 26:1487-1503

Albersmeyer J, Bock H (2008) Sensitivity generation in an adaptive BDF-method. In: Bock HG, Kostina E, Phu X, Rannacher R (eds) Modeling, simulation and optimization of complex processes: proceedings of the international conference on high performance scientific computing, March 6-10, 2006, Hanoi, Vietnam, Springer, Berlin, pp 15-24

Amaldoss W, Jain S (2005a) Conspicuous consumption and sophisticated thinking. Manag Sci 51:1449-1466

Amaldoss W, Jain S (2005b) Pricing of conspicuous goods: a competitive analysis of social effects. J Mark Res 42:30-42

Artzner P, Delbaen F, Eber J, Heath D (1999) Coherent measures of risk. Math Finance 9:203-228

Bertsimas D, Brown D, Caramanis C (2011) Theory and applications of robust optimization. SIAM Rev 53:464-501

Bock H, Plitt K (1984) A Multiple Shooting algorithm for direct solution of optimal control problems. In: Proceedings of the 9th IFAC World Congress, Pergamon Press, Budapest, pp 242-247

Caulkins J, Feichtinger G, Grass D, Hartl R, Kort P, Seidl A (2010) Two-stage conspicuous consumption model, working paper

Caulkins J, Feichtinger G, Grass D, Hartl R, Kort P, Seidl A (2011) Optimal pricing of a conspicuous product during a recession that freezes capital markets. J Econ Dyn Control 35(1):163-174

Diehl M, Bock H, Kostina E (2006) An approximation technique for robust nonlinear optimization. Math Progr 107:213-230

Diehl M, Gerhard J, Marquardt W, Moennigmann M (2008) Numerical solution approaches for robust optimal control problems. Comput Chem Eng 32:1279-1292

Heine T, Kawohl M, King R (2006) A new approach for robust optimization based open- and closed-loop control of nonlinear processes. Automatisierungstechnik 54:614-621 (in German)

Huschto T, Feichtinger G, Kort P, Hartl R, Sager S, Seidl A (2011) Numerical solution of a conspicuous consumption model with constant control delay. Automatica 47:1868-1877

Jorion P (2006) Value at risk: the new benchmark for managing financial risk. McGraw-Hill, New York

Julier S, Uhlmann JK (1996) A general method for approximating nonlinear transformations of probability distributions

Julier S, Uhlmann J (1997) A new extension of the kalman filter to nonlinear systems. Tech. rep

Kort P, Caulkins J, Hartl R, Feichtinger G (2006) Brand image and brand dilution in the fashion industry. Automatica 42:1363-1370

Kühl P, Diehl M, Milewska A, Molga E, Bock H (2007) Robust NMPC for a benchmark fed-batch reactor with runaway conditions. In: Findeisen R, Allgoewer F, Biegler L (eds) Assessment and future directions of nonlinear model predictive control. Lecture notes in control and information sciences, vol 358. Springer, Berlin, pp 455-464

Leineweber D (1999) Efficient reduced SQP methods for the optimization of chemical processes described by large sparse DAE models, Fortschritt-Berichte VDI Reihe 3, Verfahrenstechnik, vol 613. VDI Verlag, Düsseldorf

Leineweber D, Bauer I, Bock H, Schlöder J (2003) An efficient multiple shooting based reduced SQP strategy for large-scale dynamic process optimization. Part I: theoretical aspects. Comput Chem Eng 27:157-166 
Ma D, Braatz R (2001) Worst-case analysis of finite-time control policies. IEEE Trans Control Syst Technol 9:766-774

Nagy Z, Braatz R (2004) Open-loop and closed-loop robust optimal control of batch processes using distributional and worst-case analysis. J Process Control 14:411-422

Nelissen RM, Meijers MH (2011) Social benefits of luxury brands as costly signals of wealth and status. Evol Human Behav 32:343-355

Prékopa A (1995) Stochastic programming. Kluwer Academic Publishers, Norwell

Recker S, Kühl P, Diehl M, Bock HG, Marquardt W (2011) Sigmapoint approach for robust optimization of nonlinear dynamic systems. Elsevier, Amsterdam

Rockafellar R (2007) Tutorials in operations research: OR tools and applications: glimpses of future technologies, INFORMS, chap Coherent Approaches to Risk in Optimization Under Uncertainty, pp 38-61

Rockafellar R, Uryasev S (2000) Optimization of conditional value-at-risk. J Risk 2:21-42

Rockafellar R, Uryasev S (2002) Conditional value-at-risk for general loss distributions. J Bank Finance 26:1443-1471

Shapiro A, Dentcheva D, Ruszczyński A (2009) Lectures on stochastic programming: modeling and theory. In: Society for industrial mathematics 